\title{
ANGULAR DISTRIBUTION OF RADIATION EMITTED BY ARBITRARY ENSEMBLES OF NUCLEI
}

\author{
by J. A. M. COX *) and S. R. DE GROOT \\ Instituut voor theoretische Natuurkunde, Universiteit, Utrecht, Nederland
}

\section{Synopsis}

An arbitrary ensemble is described by a density matrix $\varrho$ with arbitrary values of its matrix elements. An equivalent description is given by F a n o's statistical tensors, which are used in this paper. A simple closed formula for the angular distribution of nuclear radiation from an arbitrary ensemble is derived.

$\S 1$. Introduction. An arbitrary ensemble of nuclei must be described by a density matrix,

$$
\varrho_{m m^{\prime}}=\overline{\overline{c_{m^{\prime}}^{*} c_{m}}}
$$

where the double bar indicates an ensemble average. The numbers $c_{m}$ are the coefficients of an expansion of a pure state $|A\rangle$ into basic vectors $|m\rangle$

$$
|A\rangle=\Sigma_{m} c_{m}|m\rangle \text {. }
$$

Here $m$ is the magnetic quantum number; the angular momentum of all nuclei is supposed to be $j$. In this paper we shall investigate the angular distribution of nuclear radiation emitted by an arbitrary ensemble of nuclei. This problem presents itself in the theoretical treatment of many phenomena as will be illustrated by the following considerations.

First of all, if the quantization axis has rotational symmetry a simplification is obtained since in this case the density matrix is on diagonal form. A first example of this situation is found in the problem of the angular distribution of radiation in a nuclear reaction

*) Present address: Van der Waals Laboratorium, Universiteit, Amsterdam, Nederland. 
caused by unidirectional irradiation (which direction is taken as quantization axis). A second example is the directional correlation of successive radiations, when the direction of the first radiation is taken as quantization axis ${ }^{1}$ ). A third example is the angular distribution of radiation from an ensemble of nuclei oriented in such a way that an axis of rotational symmetry exists. The angular effects of $\gamma$-radiation in the latter case have been treated in previous papers ${ }^{2}{ }^{3}$ ). In all these examples one studies the angular distribution of nuclear radiation emitted by an ensemble with cylindrical symmetry, which is prepared by preceding effects.

However, the general case with non-vanishing off-diagonal density matrix elements has also physical importance for a number of phenomena. This can easily be seen in the nuclear reaction of the type $(a, b, c)$, i.e., one incident particle $a$ in a fixed direction, followed by two successive radiations $b$ and $c$. After the absorption of particles a the nuclei form an ensemble which has no longer spherical symmetry but symmetry around the direction of a. After emission of $b$ in a fixed direction this symmetry has also disappeared and the ensemble is described by a density matrix with non-vanishing off-diagonal elements. If one wishes to calculate the angular correlation between $b$ and $c$ we have the problem of finding the angular distribution of radiation c. A second example is an ensemble of nuclei which has arisen from orientation with cylindrical symmetry, and subsequent emission of a first radiation in a fixed direction. With the density matrix of this ensemble the distribution of a second radiation can be calculated (in other words the correlation of successive radiations from oriented nuclei). Finally a third example is an ensemble of nuclei which has been oriented by means of such methods, that the system has no axis of rotational symmetry ${ }^{4}{ }^{5}{ }^{6}$ ). The calculation of the angular distribution of radiation from such an ensemble presents again the full problem of this paper.

For the calculation of the angular distribution of the radiation we shall use an alternative description of the ensemble from that given by the density matrix $\varrho_{m m^{\prime}}$. This description is provided by F a n o's statistical tensors ${ }^{7}$ ). They are defined by

$$
\langle\mid j j k q\rangle=\Sigma_{m m^{\prime}} \varrho_{m m^{\prime}}(-1)^{i-m}\left\langle j m^{\prime} j-m \mid j j k q\right\rangle .
$$

Here $\left\langle j m^{\prime} j-m \mid j j k q\right\rangle$ are the transformation coefficients for the addition of angular momenta. The set of statistical tensors $\langle\mid j j k q\rangle$ 
(with $k=0 \ldots 2 j$ and $q=-k \ldots+k$ ) is equivalent to the density matrix $\varrho_{m m^{\prime}}$. They are, however, more appropriate in considerations on effects of geometrical nature. In fact with the use of the statistical tensors we derive in section 2 a simple closed expression for the angular distribution of radiation from an arbitrary ensemble.

In section 3 we give formulae for a few special cases. In section 4 we indicate in more detail some possibilities for the application of the formulae obtained.

\$2. Derivation of the angular distribution function. If an initial state of a nucleus is denoted by $|A\rangle$ and a final state by $|f\rangle$, the relative probability of a nuclear radiation to be emitted in the direction $\mathbf{k}$ by the ensemble is given by

$$
W(\mathbf{k})=\Sigma_{i} \overline{\overline{\left.\langle A|H(\mathbf{k})| f\rangle\right|^{2}}} .
$$

The double bar indicates an average over the ensemble of nuclei. The operator $H(\mathbf{k})$ is the interaction Hamiltonian for the radiation and the nucleus. Introducing in (4) the density matrix defined by (1) and (2) we obtain

$$
W(\mathbf{k})=\Sigma_{m m^{\prime} m_{f}} \varrho_{m m^{\prime}}\left\langle j m|H(\mathbf{k})| j_{l} m_{l}\right\rangle *\left\langle j m^{\prime}|H(\mathbf{k})| j_{f} m_{f}\right\rangle .
$$

We shall use the algebra of tensor operators, developed by $\mathrm{R}$ a$c \mathrm{a} \mathrm{h}{ }^{\mathrm{B}}$ ), for the evaluation of (5). The Hamiltonian is expanded in irreducible tensor operators. In a coordinate system with $\mathbf{k}$ as $z$-axis, relative to which the emitted radiation is described, this expansion reads

$$
\bar{H}=\Sigma_{L M} \alpha_{L M} T_{M}^{L}
$$

In (6) the $\alpha_{L M}$ are parameters which characterize the emitted radiation. The components $T_{M}^{L}$ of the irreducible tensor operators of degree $L$ operate on the nucleus. Relative to the coordinate system with the quantization axis as $z$ axis (6) becomes

$$
H(\mathbf{k})=\Sigma_{L M \mu} \alpha_{L M} T_{\mu}^{L} D_{\mu M}^{L}(S),
$$

where $S$ is the rotation in space which transforms the coordinate system used in (6) into the coordinate system used in (7). $D^{L}$ is the $(2 L+1)$ dimensional irreducible representation of the rotation group. With (7) we obtain for the distribution function (5)

$W(\mathbf{k})=$

$=\Sigma \varrho_{m m^{\prime}} a_{L M}^{*} \alpha_{L^{\prime} M^{\prime}}\left\langle j m\left|T_{\mu}^{L}\right| j_{f} m_{p}\right\rangle *\left\langle j m^{\prime}\left|T_{\mu^{\prime}}^{L^{\prime}}\right| j_{j} m_{f}\right\rangle D_{\mu M}^{L^{*}}(S) D_{\mu^{\prime}, m^{\prime}}^{L}(S)$. 
With the definition of $\mathrm{R}$ a $\mathrm{c}$ a h's $V$-function

$$
\langle a a b \beta \mid a b c \gamma\rangle=(-1)^{c+\gamma}(2 c+1)^{\frac{1}{2}} V(a b c ; \alpha \beta-\gamma)
$$

and the relations

$$
\begin{aligned}
& D_{\mu M}^{L_{M}^{*}}(S) D_{\mu^{\prime} M^{\prime}}^{L^{\prime}}(S)= \\
& =\Sigma_{k \varrho \sigma}(-1)^{M-\mu}\left\langle L-\mu L^{\prime} \mu^{\prime} \mid L L^{\prime} k \varrho\right\rangle D_{\varrho \sigma}^{k}(S)\left\langle L L^{\prime} k \sigma \mid L-M L^{\prime} M^{\prime}\right\rangle, \\
& \quad\left\langle j m\left|T_{\mu}^{L}\right| j_{f} m_{f}\right\rangle=(-1)^{j+m}\left\langle i\left\|T^{L}\right\| j_{\rangle}\right\rangle V\left(j j_{f} L ;-m m_{f} \mu\right),
\end{aligned}
$$

we derive from (8)

$$
\begin{aligned}
& W(\mathbf{k})=\Sigma_{L L^{\prime}}\left\langle j\left\|T^{L}\right\| j_{j}\right\rangle^{*}\left\langle j\left\|T^{L^{\prime} \|}\right\| j_{f}\right\rangle \times \\
& \times \Sigma_{\varrho_{m m^{\prime}}} a_{L M}^{*} \alpha_{L^{\prime} M^{\prime}}(-1)^{m-m^{\prime}+M-\mu+k+e}(2 k+)^{\frac{1}{2}}\left\langle L L^{\prime} k \sigma \mid L-M L^{\prime} M^{\prime}\right\rangle \times \\
& \times D_{e \sigma}^{k}(S) V\left(L L^{\prime} k ;-\mu \mu^{\prime}-\varrho\right) V\left(j j_{f} L ;-m m_{f} \mu\right) V\left(j j_{f} L^{\prime} ;-m^{\prime} m_{f} \mu^{\prime}\right) .(12)
\end{aligned}
$$

The second summation has to be extended over $m_{t}, m, m^{\prime}, M, M^{\prime}$, $\mu, \mu^{\prime}, k, \varrho$ and $\sigma$. Summation over $\mu, \mu^{\prime}$ and $m_{f}$ with the aid of formula (41) from $\mathrm{R}$ a c a h's paper ${ }^{8}$ ) gives

$$
\begin{gathered}
W(\mathbf{k})=\Sigma_{L L^{\prime}}\left\langle j\left\|T^{L}\right\| j_{j}\right\rangle^{*}\left\langle j\left\|T^{L^{\prime}}\right\| j_{j}\right\rangle \Sigma \alpha_{L M^{\prime}}^{*} \alpha_{L^{\prime} M^{\prime}}(-1)^{L-M+k} \\
\left\langle I L^{\prime} k \sigma \mid L-M L^{\prime} M^{\prime}\right\rangle \times D_{\varrho \sigma}^{k}(S) W\left(j_{i} L^{\prime} j k ; j L\right) \Sigma_{m m^{\prime}} \varrho_{m m^{\prime}}(-1)^{j-m} \\
\left\langle j m^{\prime} j-m\right| j j \ell \varrho .
\end{gathered}
$$

The second summation is over $k, \varrho, \sigma, M$ and $M^{\prime}$. We have omitted unessential factors which only affect the normalization. The functions $W\left(j_{f} L^{\prime} j k ; j L\right)$ are the $\left.\left.\mathrm{R} \mathrm{a} \mathrm{c} \mathrm{a} \mathrm{h} \mathrm{coefficients}{ }^{8}\right){ }^{9}\right)$. With the statistical tensors $\langle\mid j j k \varrho\rangle$ defined by (3) and the abbreviation

$$
C_{k \sigma}\left(L L^{\prime}\right)=\Sigma_{M M^{\prime}}(-1)^{L-M} a_{L M^{\prime}}^{*} \alpha_{L^{\prime} M^{\prime}}\left\langle L L^{\prime} k \sigma \mid L-M L^{\prime} M^{\prime}\right\rangle,
$$

the formula for the angular distribution (13) reads

$$
\begin{aligned}
W(\mathbf{k})=\Sigma_{L L^{\prime}}\left\langle j\left\|T^{L}\right\| j_{j}\right\rangle^{*}\left\langle j\left\|T^{L^{\prime}}\right\| j_{l}\right\rangle & \Sigma_{k \rho \sigma}(-1)^{k} C_{k \sigma}\left(L L^{\prime}\right) W\left(j_{f} L^{\prime} j k ; j L\right) \times \\
& \times\left\langle\mid j j k_{\varrho}\right\rangle D_{\rho \sigma}^{k}(S) .
\end{aligned}
$$

As is seen this formula contains a sum of terms of which the factors describe the relevant physical features of the problem, viz. two physical factors $\left\langle j\left\|T^{L}\right\| j_{i}\right\rangle$ and $\left\langle j\left\|T^{L^{\prime}}\right\| j_{p}\right\rangle$ pertaining to the nuclear transition, a factor $C_{k \sigma}\left(L L^{\prime}\right)$ which characterizes the radiation, the Racah coefficient containing the total angular momenta, the statistical tensors which describe the initial orientation, and finally a function $D_{\rho o}^{k}(S)$ which depends only on the Euler angles of the rotation $S$. 
$\S 3$. Special cases. For a radiation with a single value of the total angular momentum $L$ formula (15) reads

$$
W(\mathbf{k})=\Sigma_{k \rho \sigma}(-1)^{k} C_{k \sigma}(L L) W(j, L j k ; j L)\langle\mid j j k \varrho\rangle D_{\rho o}^{k}(S),
$$

where factors now irrelevant have been omitted. For the case of cylindrical symmetry of the ensemble, the density matrix has diagonal form which means that only the statistical tensors $\langle\mid j i k 0\rangle \equiv$ $\equiv \bar{f}_{k}(j)$ subsist (cf. ${ }^{2}$ ) section 2 ). Thus a specialization of $(16)$ is obtained:

$$
W(\mathbf{k})=\Sigma_{k \sigma}(-1)^{k} C_{k \sigma}(L L) W(j j L k ; L j) \bar{f}_{k}(j) D_{0 \sigma}^{k}(S),
$$

which has been derived before ${ }^{2}$ ).

For pure $2^{L}$-pole $\gamma$-rays, of which no polarization is observed (16) gives

$$
W\left(\mathbf{k}_{1}\right)=\Sigma_{k \rho}\langle L 1 L-1 \mid L L k o\rangle W(j j L k ; L j)\langle\mid j j k \rho\rangle D_{\rho 0}^{k}(S)
$$

with only even values of $k$.

The value of $C_{k \sigma}(L L)$ for $\gamma$-rays has been used in (16) (cf. ${ }^{2}$ ) formula 84). Specialization to the case of cylindrical symmetry gives

$$
W(\mathbf{k})=\Sigma_{k}\langle L 1 L-1 \mid L L k a\rangle W\left(j_{j} L k ; L j\right) \bar{f}_{k}(j) D_{00}^{k}(S)
$$

with even values for $k . D_{00}^{k}(S)$ is proportional to the Legendre polynomial $P_{k}(\cos \vartheta)$. The result (19) has also been derived before ${ }^{2}$ ).

§ 4. Remarks on some possible applications. As has been indicated in section 1, the calculation of the angular correlation of two successive radiations from oriented nuclei presents a possibility for the application of formula (15). If the ensemble of nuclei has an axis $\eta$ of rotational symmetry the density matrix is on diagonal form which corresponds to the vanishing of all quantities $\langle\mid j j k g\rangle$ with $\rho \neq 0$. After the emission of the first radiation in the direction $\mathbf{k}_{1}$ the ensembie is described by a full set of statistical tensors $\langle\mid j j k g\rangle$ which can be calculated and depends on $\mathbf{k}_{1}$. This ensemble emits the second radiation in the direction $\mathbf{k}_{\mathbf{2}}$. For the calculation of the angular distribution of this radiation we can make use of formula (15) by substituting the calculated values of $\langle\mid j j k \varrho\rangle$. The angular dependent factor referring to the direction $\mathbf{k}_{2}$ is $D_{\rho \sigma}^{k}(T)$ where $T$ is the rotation which transforms the coordinate system with $\mathbf{k}_{2}$ as $z$ axis into that whose $z$ axis is $\eta$. A different procedure is obtained if we calculate the statistical tensors $\langle\mid j j k \varrho\rangle_{\mathbf{k}_{1}}$ describing the ensemble in a coordinate system 
whose $z$ axis if $\mathbf{k}_{1}$. The relation between $\langle\mid j j k \varrho\rangle_{\mathbf{k}_{\mathbf{1}}}$ and $\langle\mid j j k \varrho\rangle_{\eta}$ is given by

$$
\langle\mid j j k \varrho\rangle_{\mathbf{k}_{1}}=\Sigma_{\mathrm{e}}\langle\mid j j k \varrho\rangle_{\eta} D_{\boldsymbol{e} \mathbf{Q}}^{k}(S)
$$

With the use of $\langle\mid j j k \varrho\rangle_{\mathbf{k}_{2}}$ in (15) we obtain again the angular distribution for the second radiation if we take for the angle dependent factor $D_{p o}^{k}\left(S^{-1} T\right)$. The rotation $S$ is defined for $\mathbf{k}_{1}$ in the same way as $T$ for $\mathbf{k}_{2}$. The formula obtained will be a natural extension of the formula for the correlation function in the case of nuclei with random orientation. In the latter case the direction $\eta$ becomes irrelevant and only the rotation $S^{-1} T$ which transforms $\mathbf{k}_{2}$ in $\mathbf{k}_{1}$ will have physical significance.

In the field of nuclear reactions we find a possibility for application of (15) in the angular correlation between the directions of a neutron and a $\gamma$ quantum emitted in the reaction ${ }^{7} \mathrm{Li}(d, n)^{8} \mathrm{Be}^{*}(\gamma)$ $\left.{ }^{8} \mathrm{Be}{ }^{10}\right)$. The fixed direction $\eta$ of the incident deuterons will prepare an ensemble of (compound) nuclei with cylindrical symmetry. The angular correlation function can then be obtained along the same lines as indicated above.

Received 19-5-53.

\section{REFERENCES}

1) H a milton, D. R., Phys. Rev, 58 (1940) 122.

2) Tol hoek, H. A. and $C$ ox, J. A. M., Physica 1\$ (1953) 101.

3) Cox, J. A. M. and Tolhoek, H. A., Physica 19 (1953) 673.

4) A bragram, A. and Pryce, M. H. L., Nature 163 (1949) 992; Proc. phys. Soc., London, A 63 (1950) 409; Proc. roy. Soc. A 205, (1951) 135 and 336.

5) B l e a n y, B., Proc. phys. Soc., London, A 64 (1951) 315; Phil. Mag. 12 (1951) 441.

6) Simo n, A., Rose, M. E. and J a u c h, J. M., Phy's. Rev. 04 (1951) 1155.

7) F a n o, U., Nat. Bur. Stand., Washington D.C., report no. 1214, 1951.

8) R a c a h, G., Phys. Rev. (52 (1942) 438.

9) Biedenharn, L. C., Blatt, J. M. and Rose, M. E., Rev, mod. Phys. IA (1952) 249.

10) Thiri o n, J., Thèse, Strassbourg 1951. 